\title{
Dirty black holes: Symmetries at stationary non-static horizons
}

\author{
A J M Medved, Damien Martin, and Matt Visser \\ School of Mathematical and Computing Sciences \\ Victoria University of Wellington \\ PO Box 600, Wellington \\ New Zealand
}

5 March 2004; LTEX-ed October 14, 2018

\begin{abstract}
We establish that the Einstein tensor takes on a highly symmetric form near the Killing horizon of any stationary but non-static (and non-extremal) black hole spacetime. [This follows up on a recent article by the current authors, gr-qc/0402069, which considered static black holes.] Specifically, at any such Killing horizon - irrespective of the horizon geometry - the Einstein tensor block-diagonalizes into "transverse" and "parallel" blocks, and its transverse components are proportional to the transverse metric. Our findings are supported by two independent procedures; one based on the regularity of the onhorizon geometry and another that directly utilizes the elegant nature of a bifurcate Killing horizon. It is then argued that geometrical symmetries will severely constrain the matter near any Killing horizon. We also speculate on how this may be relevant to certain calculations of the black hole entropy.
\end{abstract}

gr-qc/0403026

damien.martin@mcs.vuw.ac.nz, joey.medved@mcs.vuw.ac.nz

matt.visser@mcs.vuw.ac.nz 


\section{Introduction}

There has been a longstanding belief that Bekenstein's black hole entropy 11, 2,

$$
S_{B H}=\frac{1}{4} \text { [Horizon area in Planck units], }
$$

can be explained through a process of state counting. In the standard lore, the microstates in question are presumed to arise at the level of the (yet-tobe-clarified) quantum theory of gravity 3. Notably, two prime candidates for the fundamental theory — string theory [4 and loop quantum gravity 5] - have both had some (limited) success at statistically calculating this entropy.

An alternative [but perhaps complementary] viewpoint is that $S_{B H}$ can be attributed to a classically inherited symmetry of the black hole spacetime [6]. (This symmetry typically manifests as a two-dimensional conformal field theory. ${ }^{1}$ ) Such a notion has its origins in a work by Strominger [8], where it was shown that the entropy of a three-dimensional black hole [9] is effectively controlled by symmetries that arise out of the classical property of diffeomorphism invariance [10]. It has since been a challenge to generalize this type of scenario to four (and even arbitrary) dimensional spacetimes. Furthermore, it would be preferable, ideally speaking, if the controlling symmetry is directly associated with the black hole horizon [6]. (Conversely, in the Strominger picture, diffeomorphism invariance gives rise to a conformal theory that lives at spatial infinity.)

There has indeed been substantial progress in the stated directions; in particular, the treatments by Carlip [11, 12, 13 and Solodukhin [14. Nevertheless, it remains unclear as to what is, precisely, the classical symmetry that underlies these calculations. In this regard, Carlip has suggested an "asymptotic" conformal field theory in the neighborhood of the horizon [13. (Asymptotic in the sense that the symmetry becomes exact only in the limit as the horizon is approached.)

Assuming Carlip's notion to be essentially correct (for caveats, see [15]), the current authors have proposed a fundamental explanation for this asymptotic symmetry [16. To elaborate, we have demonstrated, by purely geometrical arguments, that the Einstein tensor for any static black hole spacetime

\footnotetext{
${ }^{1}$ Two-dimensional conformal field theories are nice because the entropy is easily calculated by way of the Cardy formula [7].
} 
takes on a highly symmetrical form at the horizon. ${ }^{2}$ More specifically, the Einstein tensor block-diagonalizes into "transverse" and "parallel" blocks, and its transverse components are directly proportional to the (induced) transverse metric. ${ }^{3}$ The Einstein field equation then implies that the stress energy tensor on the static Killing horizon takes the following form:

$$
\left.T_{\nu}^{\mu}\right|_{H}=\left[\begin{array}{cc|c}
-\rho_{H} & 0 & 0 \\
0 & -\rho_{H} & 0 \\
\hline 0 & 0 & {\left[T^{\mu}{ }_{\nu}\right]_{\|}}
\end{array}\right] \text {, }
$$

where $\rho_{H}$ is the energy density at the horizon and, by virtue of symmetry, $\left[p_{\perp}\right]_{H}=-\rho_{H}$ is the transverse component of the pressure. ${ }^{4}$ In particular, we would expect, on the basis of Ehrenfest's theorem, that any contribution to the stress energy which arises from averaged quantum effects should take this form.

The purpose of the current paper is to investigate if these symmetries persist for a stationary but non-static (black hole) Killing horizon. As before, the horizon geometry is allowed to be completely general; that is, we consider "dirty" black holes [17, 19, 20]. We are able to establish these on-horizon symmetries by purely geometrical arguments; thus extending the applicable realm to include all time-reversible stationary black holes. Moreover, it can be anticipated, on physical grounds, that a dynamical black hole whose evolution rate is small [in relation to its surface gravity] will not lead to a significantly different stress-energy tensor than that allowed by equation (2).

The rest of the manuscript is organized as follows: In the next section, we start by invoking some well-known properties of stationary Killing horizons, with our main focus being on the non-static class. This allows us to constrain the black hole spacetime metric in the neighborhood of any such horizon. (These conditions are in the form of Taylor-series expansions with

\footnotetext{
${ }^{2}$ This discussion applies, strictly speaking, only to a non-extremal horizon. Unless specified otherwise, the assumption of non-extremality will always be in effect.

${ }^{3}$ Here and throughout, directional terminology — such as transverse $(\perp)$ and parallel $(\|)$ - refers to the orientation of a (typically arbitrary) spacelike cross-section of the horizon.

${ }^{4}$ This basic result has been known for some time for a static spherically symmetric Killing horizon [17] 18. The novelty of our recent work [16] was to lift the stringent condition of spherical symmetry. For instance, an asymmetric "ring" of material placed around the equator of a Schwarzschild-like black hole would distort the horizon from a spherical into an ovaloid shape.
} 
respect to the normal distance from the horizon.) We are able to substantiate these constraints, in Section 3, by appealing to the regularity of curvature invariants on the horizon. Then, in Section 4, after outlining the relevant methodology, we finally present the on-horizon form of the Einstein tensor. Section 5 provides an alternative argument as to why any [bifurcate] stationary Killing horizon must have a Einstein tensor with the advertised symmetries. We conclude in Section 6 with a discussion.

\section{Boundary conditions at the horizon}

First of all, the following observation will allow us to greatly simplify the problem at hand: Stationary black holes are expected to be either static or axially symmetric. (See, for instance, page 523 of [21.) To better understand this notion, let us consider some black hole that is rotating. If such a black hole is embedded in a spacetime that is not axially symmetric, there will be a tidal frictional force that acts to both slow down the rotation and smooth out the axial asymmetry. Consequently, such a spacetime cannot be stationary; rather, it must still be evolving. However, once the evolution has finally stopped, there can only be two possibilities:

- The rotation has completely stopped, in which case the black hole is static but not necessarily axially symmetric.

- The black hole is still rotating, but all of the axial asymmetries have been smoothed out so that there is no longer any tidal friction. In this case, the black hole is stationary, non-static and axially symmetric.

Considering that the static case has already been covered in our prior work [16], we will now assume an axially symmetric spacetime (until the more general analysis of Section 5).

Since the spacetime in question has been deemed as axially symmetric, there must be a rotation axis that picks out a particular spacelike direction say $\phi-$ and two independent Killing vectors: a timelike one, $\psi^{\mu}=\left[\partial_{t}\right]^{\mu}$, and an axial one, $\xi^{\mu}=\left[\partial_{\phi}\right]^{\mu}$. It is, therefore, possible to introduce coordinates $\left(t, \phi, x^{2}, x^{3}\right)$ such that

$$
g_{\mu \nu}=\left[\begin{array}{cccc}
g_{t t} & g_{\phi t} & g_{t 2} & g_{t 3} \\
g_{\phi t} & g_{\phi \phi} & g_{\phi 2} & g_{\phi 3} \\
g_{t 2} & g_{\phi 2} & g_{22} & g_{23} \\
g_{t 3} & g_{\phi 3} & g_{23} & g_{33}
\end{array}\right]
$$


where the metric components are functions of the remaining spacelike coordinates, $x^{2}$ and $x^{3}$, only.

Let us now impose the additional constraint that our spacetime is invariant under "time-reversal", which means the spacetime is invariant under the simultaneous change of

$$
t \rightarrow-t \quad \text { and } \quad \phi \rightarrow-\phi
$$

(That is, a change in the direction of time should correspond to a reverse in the sense of rotation.) As a consequence, the above metric can be simplified as follows ( $c f$, Section 7.1 of [21]):

$$
g_{\mu \nu}=\left[\begin{array}{cccc}
g_{t t} & g_{\phi t} & 0 & 0 \\
g_{\phi t} & g_{\phi \phi} & 0 & 0 \\
0 & 0 & g_{22} & g_{23} \\
0 & 0 & g_{23} & g_{33}
\end{array}\right]
$$

The existence of such a symmetry is, under certain circumstances, a theorem that can be derived from integrability conditions placed on the Killing vectors. However, we feel that most physicists would be happy to simply assume the symmetry on physical grounds.

It is convenient to transform the $2 \times 2$-block in the $t-\phi$-plane into an ADM-like form; namely,

$$
g_{\mu \nu}=\left[\begin{array}{cccc}
-\left[N^{2}-g_{\phi t}^{2} / g_{\phi \phi}\right] & g_{\phi t} & 0 & 0 \\
g_{\phi t} & g_{\phi \phi} & 0 & 0 \\
0 & 0 & g_{22} & g_{23} \\
0 & 0 & g_{23} & g_{33}
\end{array}\right]
$$

where $N$ denotes the usual "lapse" function. This formulation makes it clear that the ergosurface of the black hole is located at

$$
g_{t t}=0 \Longleftrightarrow N^{2}=g_{\phi t}^{2} / g_{\phi \phi},
$$

while the horizon (or surface of infinite red-shift) is at

$$
N=0
$$

To proceed, it proves useful if the $2 \times 2$-block in the $x^{2}-x^{3}$-plane is appropriately simplified. Let us call these two coordinates $n$ and $z$; with $n$ 
representing the normal distance to the horizon (such that $N=0$ at $n=0$ ) and the $z$-direction being perpendicular to that of $n$. Then, without any loss of generality, we have

$$
g_{\mu \nu}=\left[\begin{array}{cccc}
-\left[N^{2}-g_{\phi t}^{2} / g_{\phi \phi}\right] & g_{\phi t} & 0 & 0 \\
g_{\phi t} & g_{\phi \phi} & 0 & 0 \\
0 & 0 & 1 & 0 \\
0 & 0 & 0 & g_{z z}
\end{array}\right]
$$

where $N, g_{\phi \phi}, g_{\phi t}$ and $g_{z z}$ can now be regarded as functions of $n$ and $z$. Such Gaussian normal coordinates can only be expected to have validity for some region around the $n=0$ surface, insofar as the geodesics defining our coordinate system will typically intersect. This will, however, not be an issue in the analysis, as we are always looking at the near-horizon limit.

It is also convenient to define an "angular-rotation" parameter such that $\omega \equiv-g_{\phi t} / g_{\phi \phi}$, and so

$$
g_{\mu \nu}=\left[\begin{array}{cccc}
-\left[N^{2}-g_{\phi \phi} \omega^{2}\right] & -g_{\phi \phi} \omega & 0 & 0 \\
-g_{\phi \phi} \omega & g_{\phi \phi} & 0 & 0 \\
0 & 0 & 1 & 0 \\
0 & 0 & 0 & g_{z z}
\end{array}\right]
$$

or, equivalently,

$$
\mathrm{d} s^{2}=-N(n, z)^{2} \mathrm{~d} t^{2}+g_{\phi \phi}(n, z)\{\mathrm{d} \phi-\omega(n, z) \mathrm{d} t\}^{2}+\mathrm{d} n^{2}+g_{z z}(n, z) \mathrm{d} z^{2} .
$$

We can be even more specific about the metric by considering some wellknown properties of stationary Killing horizons. Firstly, there is a zeroth law of black hole mechanics [21, 22, which tells us that the surface gravity or

$$
\kappa_{H} \equiv \lim _{n \rightarrow 0} \partial_{n} N
$$

must be a non-negative constant on the horizon. ${ }^{5}$ Note that current considerations will be restricted to non-extremal horizons, for which $\kappa_{H}>0 .{ }^{6}$

\footnotetext{
${ }^{5}$ Following an analysis which is very similar to that of the appendix in [16], one can readily verify that equation (12) complies with the standard definition of the surface gravity 21].

${ }^{6}$ For the static case, we have previously shown that an extremal horizon $\left(\kappa_{H}=0\right)$ must be located at an infinite proper distance, $n=-\infty$ [16. As briefly discussed in Section 3, similar arguments lead to the same conclusion for any stationary black hole.
} 
Secondly, there is a rigidity theorem for axially symmetric (stationary, nonstatic) Killing horizons [23], which states that

$$
\Omega_{H} \equiv-\lim _{n \rightarrow 0} \frac{g_{\phi t}}{g_{\phi \phi}}=\lim _{n \rightarrow 0} \omega
$$

is also a constant on the horizon. Finally, let us take note of the following observation made by Carter [see [23], equation (6.95)]: A stationary Killing horizon is a geodesic submanifold; so that, if one starts at any point in the horizon and follows the geodesic along any tangent vector to the horizon, the resultant curve must then remain in the horizon. This implies that the horizon is extrinsically flat [see [23], equation (6.124)]; meaning that the extrinsic curvature of the horizon, itself, must be zero. Hence, ${ }^{7}$

$$
\lim _{n \rightarrow 0} K_{\mu \nu}=-\frac{1}{2} \lim _{n \rightarrow 0} \frac{\partial g_{\mu \nu}}{\partial n}=0 .
$$

The above properties directly imply a set of necessary constraints, which can be expressed in terms of Taylor-series expansions as follows:

$$
\begin{gathered}
g_{\phi \phi}(n, z)=\left[g_{H}\right]_{\phi \phi}(z)+\frac{1}{2}\left[g_{2}\right]_{\phi \phi}(z) n^{2}+o\left(n^{3}\right), \\
g_{z z}(n, z)=\left[g_{H}\right]_{z z}(z)+\frac{1}{2}\left[g_{2}\right]_{z z}(z) n^{2}+o\left(n^{3}\right), \\
\omega(n, z)=\Omega_{H}+\omega_{1}(z) n+\frac{1}{2} \omega_{2}(z) n^{2}+o\left(n^{3}\right),
\end{gathered}
$$

and

$$
N(n, z)=\kappa_{H} n+\frac{1}{2} \kappa_{1}(z) n^{2}+\frac{1}{3 !} \kappa_{2}(z) n^{3}+o\left(n^{4}\right)
$$

In analogy with the static case, one might expect to be able to derive the stronger result [16]

$$
N(n, z)=\kappa_{H} n+\frac{1}{3 !} \kappa_{2}(z) n^{3}+o\left(n^{4}\right)
$$

so as to avoid a curvature singularity on the horizon. Although true, we cannot conclude this from the current argument since $\kappa_{1}(z)$ could still be a function of $\omega$ that vanishes as $\omega \rightarrow 0$. In the next section, we explicitly write out the Taylor series and demonstrate that equation (19) is indeed necessary. Similarly, it is also shown that the on-horizon extrinsic curvature and the coefficient $\omega_{1}(z)$ are required to vanish.

\footnotetext{
${ }^{7}$ Note that we use Misner-Thorne-Wheeler conventions for the extrinsic curvature. In particular, see page 552 of 24 .
} 


\section{Boundary conditions revisited}

Next, we will be able to explicitly verify the constraint equations (15) and (16), strengthen (17) and substantiate (19) by requiring on-horizon regularity. More precisely, we will require a well-behaved horizon 2-geometry and the absence of curvature singularities on the horizon. Note that any of the following calculations can be obtained by a somewhat tedious hand calculation, although we have, at times, opted for a symbolic computation using Maple.

We begin here by recalling the metric of equation (11), as appropriate for the near-horizon geometry of a stationary Killing horizon with axial (and time-reversal) symmetry. Keep in mind that the horizon is located, by construction, at the surface where $n=0$. (Note that this formalism can, strictly speaking, only be valid for a non-extremal horizon, as an extremal horizon cannot occur at a finite value of $n$ [25]. Later on, when we look explicitly at extremal horizons, this well-known fact will drop out quite naturally.)

For calculational purposes, let us expand the lapse and the rotation parameter in the most general manner possible [subject only to the constraint that the lapse vanishes on the horizon]; that is,

$$
\begin{gathered}
N(n, z)=\kappa_{H}(z) n+\frac{1}{2} \kappa_{1}(z) n^{2}+\frac{1}{3 !} \kappa_{2}(z) n^{3}+O\left(n^{4}\right), \\
\omega(n, z)=\omega_{H}(z)+\omega_{1}(z) n+\frac{1}{2} \omega_{2}(z) n^{2}+\frac{1}{3 !} \omega_{3}(z) n^{3}+O\left(n^{4}\right) .
\end{gathered}
$$

The following point should be emphasized: We are not assuming our refined form for the lapse $[c f$, equation (19)] but, rather, attempting to verify this result (along with the other refinements) by independent means.

Essentially, we are interested in the regularity (or lack thereof) of the following curvature invariants at the horizon:

- The Ricci scalar, $R$.

- The traceless part of the Ricci tensor squared, $R_{\mu \nu} R^{\mu \nu}-\frac{1}{4} R^{2}$.

- The Weyl tensor squared.

Clearly, if any of these three scalars are infinite at the horizon, then a curvature singularity exists. 
First, a calculation of the Ricci scalar yields

$$
R=\frac{\left[g_{H}\right]_{\phi \phi}(z)}{2\left[g_{H}\right]_{z z}(z) \kappa_{H}(z)^{2}}\left\{\left[g_{H}\right]_{z z} \omega_{1}(z)^{2}+\left(\frac{\mathrm{d} \omega_{H}(z)}{\mathrm{d} z}\right)^{2}\right\} \frac{1}{n^{2}}+o\left(\frac{1}{n}\right) .
$$

[Note that, since the horizon 2-geometry is assumed to be regular, $g_{z z}$ must be positive on the horizon.] Now, to avoid a curvature singularity, the coefficient of any negative power of $n$ must be zero. Given that the leading-order term is a sum of squares, we immediately obtain two conditions:

$$
\begin{aligned}
\frac{\mathrm{d} \omega_{H}(z)}{\mathrm{d} z} & =0 \Rightarrow \omega_{H}(z)=\omega_{H}=\text { constant }, \\
\omega_{1}(z) & =0 .
\end{aligned}
$$

Hence, we have recovered both the rigidity theorem [23] and the anticipated vanishing of the linear-order term.

Second, let us consider the traceless part of the Ricci tensor squared. Using the previous results to simplify matters, we find that

$$
\begin{aligned}
4 R_{\mu \nu} R^{\mu \nu}-R^{2}= & \left\{\left(\left.\frac{\mathrm{d} \ln g_{z z}}{\mathrm{~d} z}\right|_{n=0}\right)^{2}+\left(\left.\frac{\mathrm{d} \ln g_{\phi \phi}}{\mathrm{d} z}\right|_{n=0}\right)^{2}\right. \\
& \left.+\frac{16 \kappa_{1}(z)^{2}}{\kappa_{H}(z)^{2}}+\frac{8\left(\mathrm{~d} \kappa_{H}(z) / \mathrm{d} z\right)^{2}}{\left[g_{H}\right]_{z z}(z) \kappa_{H}(z)}\right\} \frac{1}{n^{2}}+o\left(\frac{1}{n}\right) .
\end{aligned}
$$

This is, once again, a sum of squares, from which we can deduce the following four constraints:

$$
\begin{aligned}
\frac{\mathrm{d} \kappa_{H}(z)}{\mathrm{d} z} & =0 \quad \Rightarrow \quad \kappa_{H}(z)=\kappa_{H}=\text { constant } \\
\kappa_{1}(z) & =0 \\
\left.\frac{\mathrm{d} g_{z z}}{\mathrm{~d} z}\right|_{n=0} & =0 \Rightarrow g_{z z}=\left[g_{z z}(z)\right]_{H}+o\left(n^{2}\right), \\
\left.\frac{\mathrm{d} g_{\phi \phi}}{\mathrm{d} z}\right|_{n=0} & =0 \quad \Rightarrow \quad g_{\phi \phi}=\left[g_{\phi \phi}(z)\right]_{H}+o\left(n^{2}\right) .
\end{aligned}
$$

Note that the first of these conditions allows us to recover the zeroth law 21 , 22 .

Finally, after imposing all the above constraints, we find that the Weyl tensor squared yields a finite result as $n \rightarrow 0$ [i.e., the leading order is at 
least $o(1)]$. Therefore, our regularity requirements leave us with a set of six constraints; ${ }^{8}$ all of which are necessary and, in combination, sufficient for the horizon not to have any curvature singularities. In this unambiguous way, we have substantiated the series expansions in equations (15)-(17) [with $\omega_{1}=0$ ] and equation (19). Moreover, the above statements can be regarded as a rigorous proof, which supersedes our earlier plausibility arguments.

Let us now address the issue of extremal horizons, for which $\kappa_{H}=0$. Assuming an $m$-order degeneracy in the surface gravity, we can expand the lapse as

$$
N(n, z)=\frac{\kappa_{m}(z)}{m !} n^{m}+\frac{\kappa_{m+1}(z)}{(m+1) !} n^{m+1}+o\left(n^{m+2}\right) .
$$

Now proceeding just as before, we find that,

$$
R=\frac{\left[g_{H}\right]_{\phi \phi} m !\left\{\left[g_{H}\right]_{z z} \omega_{1}(z)^{2}+\left(\mathrm{d} \omega_{0}(z) / \mathrm{d} z\right)^{2}\right\}}{2\left[g_{H}\right]_{z z} \kappa_{m}(z)^{2}} n^{-2 m}+o\left(n^{-2 m+1}\right),
$$

which informs us that the rigidity theorem still holds and the linear term $\omega_{1}$ vanishes. Working through the subdominant terms, we can place still more constraints on $\omega(n, z)$. Then, after some effort, we arrive at

$$
R_{\mu \nu} R^{\mu \nu}=\frac{m^{2}(m-1)^{2}}{n^{4}}+\frac{1}{4} \frac{\left[g_{H}\right]_{\phi \phi}^{2} m^{4}}{n^{4}\left[g_{H}\right]_{z z}^{2} \kappa_{m}(z)^{4}}\left(\frac{\mathrm{d} \omega_{m-1}(z)}{\mathrm{d} z}\right)^{4}+o\left(\frac{1}{n^{3}}\right)
$$

which is a sum of squares. Because the first term is required to vanish, either $m=1$ (a non-extremal horizon at $n=0$ ) or $m=0$ (no horizon at $n=0)$. Since any finite value of $n$ can always be shifted to $n=0$, this really tells us that an extremal horizon cannot occur at a finite value of the normal coordinate. Hence, as in the static case, any extremal horizon must be located at $n=-\infty$.

\section{The on-horizon Einstein tensor}

We can now, with the help of a symbolic Maple computation, use these Taylor-series expansions to deduce the various components of the on-horizon Einstein tensor. However, to express this tensor in a form that emphasizes

\footnotetext{
${ }^{8}$ One can use the above conditions to verify that no further constraints can be extracted from the $o\left(n^{-1}\right)$ terms in equations (22) and (25).
} 
its symmetrical nature, it will first be necessary to introduce some additional formalism.

To begin, let us take note of the null Killing vector for a stationary (black hole) Killing horizon. That is (for instance, [21]),

$$
\chi^{a}=\xi^{a}+\Omega_{H} \psi^{a} .
$$

Then, in terms of our $(t, \phi, n, z)$ coordinate system, we have

$$
\begin{gathered}
\chi^{a}=\left(1, \Omega_{H}, 0,0\right), \\
\xi^{a}=(1,0,0,0),
\end{gathered}
$$

and

$$
\psi^{a}=(0,1,0,0) .
$$

Let us next consider the contraction

$$
g(\chi, \chi) \equiv g_{a b} \chi^{a} \chi^{b}=g_{t t}+2 \Omega_{H} g_{t \phi}+\Omega_{H}^{2} g_{\phi \phi}=-N^{2}+g_{\phi \phi}\left[\Omega_{H}-\omega\right]^{2},
$$

which, by an inspection of our Taylor-series expansions, implies that

$$
g(\chi, \chi)=-\kappa_{H}^{2} n^{2}+o\left(n^{4}\right) .
$$

It would then seem sensible to define, outside of the event horizon, a normalized vector of the form

$$
\hat{\chi}=\frac{\chi}{\sqrt{-g(\chi, \chi)}} .
$$

We can similarly write

$$
g(\psi, \psi)=g_{\phi \phi}=o(1)
$$

and

$$
\hat{\psi}=\frac{\psi}{\sqrt{g(\psi, \psi)}}=\frac{\psi}{\sqrt{g_{\phi \phi}}}
$$

Furthermore,

$$
g(\chi, \psi)=g_{a b} \chi^{a} \psi^{b}=g_{t \psi}+\Omega_{H} g_{\psi \psi}=g_{\psi \psi}\left(\Omega_{H}-\omega\right)=o\left(n^{2}\right)
$$

and

$$
g(\hat{\chi}, \hat{\psi})=o(n)
$$


Finally,

$$
\hat{n}=n=(0,0,1,0)
$$

and

$$
\hat{z}=\frac{(0,0,0,1)}{\sqrt{g_{z z}}} .
$$

Granted, $\hat{\chi}$ and $\hat{\psi}$ are not exactly orthonormal. But these vectors are certainly orthonormal to the order $o(n)$ and span the space perpendicular to the horizon, which is enough to investigate the on-horizon geometry.

We are now suitably positioned to discuss the on-horizon components of the Einstein tensor. For this purpose, it is helpful to define

$$
\begin{aligned}
{\left[G_{\hat{\chi} \hat{\chi}}\right]_{H} \equiv } & \lim _{n \rightarrow 0} G(\hat{\chi}, \hat{\chi})=\lim _{n \rightarrow 0} \frac{G(\chi, \chi)}{g(\chi, \chi)} \\
& =\lim _{n \rightarrow 0} \frac{G_{t t}+2 \Omega_{H} G_{t \phi}+\Omega_{H}^{2} G_{\phi \phi}}{\kappa_{H}^{2} n^{2}} \\
{\left[G_{n n}\right]_{H} \equiv } & \lim _{n \rightarrow 0} G(n, n)=\lim _{n \rightarrow 0} G_{n n} \\
{\left[G_{\hat{z} \hat{z}}\right]_{H} \equiv } & \lim _{n \rightarrow 0} G(\hat{z}, \hat{z})=\lim _{n \rightarrow 0} \frac{G_{z z}}{g_{z z}} \\
{\left[G_{\hat{\phi} \hat{\phi}}\right]_{H} \equiv } & \lim _{n \rightarrow 0} G(\hat{\psi}, \hat{\psi})=\lim _{n \rightarrow 0} \frac{G(\psi, \psi)}{g(\psi, \psi)}=\lim _{n \rightarrow 0} \frac{G_{\phi \phi}}{g_{\phi \phi}} \\
{\left[G_{\hat{\phi} \hat{\chi}}\right]_{H} \equiv } & \lim _{n \rightarrow 0} G(\hat{\chi}, \hat{\psi})=\lim _{n \rightarrow 0} \frac{G(\chi, \psi)}{\sqrt{g(\chi, \chi) g(\psi, \psi)}} \\
{\left[G_{n \hat{z}}\right]_{H} \equiv } & \lim _{n \rightarrow 0} G(n, \hat{z})=\lim _{n \rightarrow 0} \frac{G_{n z}}{\sqrt{g_{z z}}},
\end{aligned}
$$

with all other components automatically vanishing by virtue of the axial symmetry and/or time-reversal symmetry.

Ideally speaking, what we would now like to prove is the following:

$$
\begin{gathered}
{\left[G_{\hat{\chi} \hat{\chi}}\right]_{H}=-\left[G_{n n}\right]_{H}} \\
{\left[G_{\hat{\phi} \hat{\chi}}\right]_{H}=0}
\end{gathered}
$$

and

$$
\left[G_{n \hat{z}}\right]_{H}=0
$$


Such an outcome would confirm that, just as for a static Killing horizon [16], the Einstein tensor block-diagonalizes and the transverse components of this tensor are proportional to the transverse metric. (By transverse, we mean the components orthogonal to any spacelike cross-section of the event horizon.) Before elaborating further on our results, let us point out that there is, in fact, a strong physical motivation for believing in the vanishing of $\left[G_{\hat{\phi} \hat{\chi}}\right]_{H}$. By the Einstein equations, this term is equivalent to a non-zero angular "flux" at the horizon. Significantly, the null Killing vector, $\chi$, effectively "rotates with the horizon". Hence, if any such flux does exist, the implication would be that the dirt surrounding the black hole is "moving with respect to the horizon". But this would then torque the black hole - either spinning it up or slowing it down - until the true state of stationarity is finally achieved. (Alas, we have no analogously simple argument for the vanishing of the "stress" term $\left.\left[G_{n \hat{z}}\right]_{H} \cdot\right)$

All three of these symmetries have indeed been verified by a symbolic computation. To briefly elaborate, using our Taylor-series expansions [i.e., equations (15)-(17) with $\omega_{1}=0$ and (19)], we "ask" Maple to calculate the Einstein tensor and, afterwards, take the $n \rightarrow 0$ limit. A simple inspection then confirms the validity of equations (152)-(154).

For the sake of completeness, we will also present the explicit form of these on-horizon tensor components. It is useful, however, if we first introduce a few more relevant expressions. For instance, considering just the in-horizon 2-metric or

$$
\mathrm{d} s_{\|}^{2}=g_{\phi \phi}(z) \mathrm{d} \phi^{2}+g_{z z}(z) \mathrm{d} z^{2},
$$

we can calculate the corresponding scalar curvature and obtain

$$
R_{\|}=\frac{1}{2}\left\{\frac{\left(\partial_{z}\left[g_{H}\right]_{\phi \phi}\right)^{2}}{\left[g_{H}\right]_{z z}\left[g_{H}\right]_{\phi \phi}^{2}}+\frac{\partial_{z}\left[g_{H}\right]_{\phi \phi} \partial_{z}\left[g_{H}\right]_{z z}}{\left[g_{H}\right]_{z z}^{2}\left[g_{H}\right]_{\phi \phi}}-2 \frac{\partial_{z}^{2}\left[g_{H}\right]_{\phi \phi}}{\left[g_{H}\right]_{z z}\left[g_{H}\right]_{\phi \phi}}\right\} .
$$

Similarly, focusing on the $\hat{\chi}^{-} n$-plane and looking at the induced transverse 2-metric,

$$
\mathrm{d} s_{\perp}^{2}=-\left[N^{2}-g_{\phi \phi}\left(\omega-\Omega_{H}\right)^{2}\right] \mathrm{d} \tilde{\chi}^{2}+\mathrm{d} n^{2},
$$

we find a corresponding Ricci scalar of the simple form

$$
R_{\perp}=-2\left\{\frac{\kappa_{2}}{\kappa_{H}}\right\}+\frac{3}{2} \frac{\left[g_{H}\right]_{\phi \phi}(z) \omega_{2}(z)^{2}}{\kappa_{H}^{2}} .
$$

It should be noted that, in obtaining this last result, we had to expand $g(\chi, \chi)$ out to the fourth order in $n$ [so that equation (38) is insufficient], as 
non-trivial contributions occur at this order as a consequence of the normal derivatives.

In terms of the above formalism, the non-vanishing components of the on-horizon Einstein tensor are now expressible as follows:

$$
\begin{aligned}
{\left[G_{\hat{\chi} \hat{\chi} \hat{\chi}}\right]_{H} } & =-\frac{1}{2} R_{\|}-\frac{1}{2} \operatorname{tr}\left[g_{2}\right]-\frac{1}{4}\left[g_{H}\right]_{\phi \phi} \frac{\omega_{2}^{2}}{\kappa_{H}^{2}}, \\
{\left[G_{n n}\right]_{H} } & =-\left[G_{\hat{\chi} \hat{\chi}}\right]_{H}, \\
{\left[G_{\hat{z} \hat{z}}\right]_{H} } & =-\frac{1}{2} R_{\perp}+\frac{\left[g_{2}\right]_{\phi \phi}}{\left[g_{H}\right]_{\phi \phi}}+\frac{1}{2}\left[g_{H}\right]_{\phi \phi} \frac{\omega_{2}^{2}}{\kappa_{H}^{2}}, \\
{\left[G_{\hat{\phi} \hat{\phi}}\right]_{H} } & =-\frac{1}{2} R_{\perp}+\frac{\left[g_{2}\right]_{z z}}{\left[g_{H}\right]_{z z}},
\end{aligned}
$$

where the trace operation (depicted by tr in the first equation) has been performed with the in-horizon 2-metric defined by equation (55).

It is easily confirmed that the above results correctly limit to their static analogues $(c f,[16])$ as $\omega \rightarrow 0$. In spite of the obvious complexities that arise for a stationary but non-static horizon, the current case is still, in some sense, a simplification from the most general static formalism. This is because the property of axial symmetry now implies that $g_{\|}$(and, hence, $\left[g_{2}\right]_{\|}$ in particular) is diagonal in the $\phi-z$-coordinates. Thus, $G_{\|}$is automatically diagonal, which was not necessarily true in the static case. On the other hand, there are now extra contributions from $\omega_{2}$. Note that $\Omega_{H}$ drops out of the on-horizon Einstein tensor completely - this can be viewed as a side effect of having a rigidly rotating horizon.

\section{An alternative method: Bifurcate Killing horizons}

It has now been confirmed that the anticipated symmetries in the Einstein tensor [namely, equations (52)-(54)] are valid at any stationary (nonextremal) Killing horizon; but what is still lacking is a clear physical motivation for this phenomenon. Here, we will help fill this gap by providing a relatively simple and physically compelling argument for this highly symmetric form. Although the upcoming analysis applies irrespective of axial symmetry, there is one important caveat: We will now restrict considerations to bifurcate Killing horizons. Which is to say, it will now be assumed 
that the horizon contains a bifurcation surface - that is, a cross-sectional (spacelike) 2-surface where the null Killing vector, $\chi^{a}$, is precisely vanishing. At a first glance, this may appear to be a highly restrictive constraint on the spacetime; in particular, a physical black hole that forms from stellar collapse will not be of this type. Nonetheless, a physical black hole will asymptotically approach such a spacetime. Indeed, Racz and Wald have shown that, if the surface gravity is constant and non-vanishing over a patch of Killing horizon (containing a spacelike cross-section), there will exist a stationary extension of the spacetime which does include a regular bifurcation surface 26 . Since the zeroth law is automatically satisfied for any stationary Killing horizon [22], the existence of such an extension will, for our purposes, always be ensured.

For the analysis of this section, it proves to be convenient if we employ a different basis for the (on-horizon) coordinate system. To set up a suitable basis, let us start by considering an arbitrary spacelike section of the horizon. Like all spacelike 2-surfaces, it is possible - at every point - to find two null vectors that are orthogonal to the section, as well as to each other. Let us choose one of these to be the Killing vector $\chi^{a}$ and denote the other by $N^{a}$. For the sake of convenience, we will adopt the normalization $\chi^{a} N_{a}=-1$. (And since both of these are null, $\chi^{a} \chi_{a}=N^{a} N_{a}=0$.)

To complete our coordinate basis, we can choose any pair of orthogonal spacelike vectors, $m_{1}^{a}$ and $m_{2}^{a}$, that are tangent to the horizon section in question. It should be clear that, by construction, $\chi^{a}, N^{a}, m_{1}^{a}$, and $m_{2}^{a}$ form an orthonormal basis for the tangent space. Consequently, there will exist coefficients such that the Einstein tensor can be written as follows:

$$
\begin{aligned}
G_{a b}= & G_{++} \chi_{a} \chi_{b}+G_{--} N_{a} N_{b}+G_{+-}\left\{\chi_{a} N_{b}+N_{a} \chi_{b}\right\} \\
& +G_{+1}\left\{\chi_{a}\left[m_{1}\right]_{b}+\left[m_{1}\right]_{a} \chi_{b}\right\}+G_{+2}\left\{\chi_{a}\left[m_{2}\right]_{b}+\left[m_{2}\right]_{a} \chi_{b}\right\} \\
& +G_{-1}\left\{N_{a}\left[m_{1}\right]_{b}+\left[m_{1}\right]_{a} N_{b}\right\}+G_{-2}\left\{N_{a}\left[m_{2}\right]_{b}+\left[m_{2}\right]_{a} N_{b}\right\} \\
& +G_{11}\left[m_{1}\right]_{a}\left[m_{1}\right]_{b}+G_{22}\left[m_{2}\right]_{a}\left[m_{2}\right]_{b} \\
& +G_{12}\left\{\left[m_{1}\right]_{a}\left[m_{2}\right]_{b}+\left[m_{2}\right]_{a}\left[m_{1}\right]_{b}\right\}
\end{aligned}
$$

(With an analogous form, in fact, for any symmetric tensor.)

We can significantly simplify the above expression by, firstly, taking note of equation (7.1.15) from Wald's textbook [21] (re-expressed in one-form notation),

$$
\chi \wedge(R \cdot \chi)=0
$$


which is valid [on the horizon] for any stationary Killing horizon. (Here, $R$ represents the two-form Ricci tensor rather than the scalar curvature.) It immediately follows that, on the horizon,

$$
(R \cdot \chi) \propto \chi
$$

or, equivalently,

$$
R_{a}^{b} \chi_{b} \propto \chi_{a} .
$$

But, since $g^{b}{ }_{a} \chi_{b}=\chi_{a}$, this also means that

$$
G^{b}{ }_{a} \chi_{b} \propto \chi_{a}
$$

and so the Einstein tensor (like the Ricci tensor) possesses a null eigenvector on the horizon.

To make use of this last property, let us contract equation (63) with $\chi^{b}$ and then apply the orthogonality properties. This process yields

$$
G_{a b} \chi^{b}=-G_{--} N_{a}-G_{+-} \chi_{a}-G_{-1}\left[m_{1}\right]_{a}-G_{-2}\left[m_{2}\right]_{a} .
$$

However, we know that, on the horizon, this contraction must be proportional to $\chi_{a}$; thus implying

$$
G_{--}=G_{-1}=G_{-2}=0,
$$

so that the on-horizon Einstein tensor reduces to

$$
\begin{aligned}
G_{a b}= & G_{++} \chi_{a} \chi_{b}+G_{+-}\left\{\chi_{a} N_{b}+N_{a} \chi_{b}\right\} \\
& +G_{+1}\left\{\chi_{a}\left[m_{1}\right]_{b}+\left[m_{1}\right]_{a} \chi_{b}\right\}+G_{+2}\left\{\chi_{a}\left[m_{2}\right]_{b}+\left[m_{2}\right]_{a} \chi_{b}\right\} \\
& +G_{11}\left[m_{1}\right]_{a}\left[m_{1}\right]_{b}+G_{22}\left[m_{2}\right]_{a}\left[m_{2}\right]_{b} \\
& +G_{12}\left\{\left[m_{1}\right]_{a}\left[m_{2}\right]_{b}+\left[m_{2}\right]_{a}\left[m_{1}\right]_{b}\right\} .
\end{aligned}
$$

This is as far as we can go on an arbitrary section of the horizon, so let us now specialize to the bifurcation surface. First note that

$$
\left[g_{\perp}\right]_{a b}=\chi_{a} N_{b}+N_{a} \chi_{b}
$$

has a well-defined limit on the bifurcation 2-surface, even though $\chi^{a} \rightarrow 0$ as the surface is approached. This is because the second null normal limits there as $N^{a} \rightarrow \infty$, since it still must satisfy $\chi^{a} N_{a}=-1$. It follows that the above combination simply limits to the 2-metric perpendicular to the 
bifurcation surface. In view of this observation, the Einstein tensor at the bifurcation 2-surface takes on the greatly simplified form

$$
\begin{aligned}
G_{a b}= & G_{+-}\left[g_{\perp}\right]_{a b} \\
& +G_{11}\left[m_{1}\right]_{a}\left[m_{1}\right]_{b}+G_{22}\left[m_{2}\right]_{a}\left[m_{2}\right]_{b} \\
& +G_{12}\left\{\left[m_{1}\right]_{a}\left[m_{2}\right]_{b}+\left[m_{2}\right]_{a}\left[m_{1}\right]_{b}\right\}
\end{aligned}
$$

or, in perhaps more sensible notation,

$$
G_{a b}=G_{+-}\left[g_{\perp}\right]_{a b}+\left[G_{\|}\right]_{a b} .
$$

Notice that, on the bifurcation surface, $G_{a b}$ indeed block-diagonalizes and its transverse components are proportional to the transverse metric. This is precisely the form of Einstein tensor we have been setting out to show! We can now use the fact that the Einstein tensor is a Killing invariant to Lie propagate this form away from the bifurcation surface and, then, onto any other spacelike section of the horizon. (See, for instance, a relevant discussion in [27.) In this way, we are able to substantiate the highly symmetric form of the on-horizon Einstein tensor, but with a completely independent and (perhaps) physically more translucent method. ${ }^{9}$

\section{Discussion}

Let us now briefly summarize the findings of this paper (in conjunction with our prior treatment [16]): We have been able to establish a very high degree of symmetry in the Einstein tensor near any stationary (static or non-static) non-extremal Killing horizon. In particular, the on-horizon form of this tensor will block-diagonalize, and its transverse components will be directly proportional to the transverse metric. As a direct consequence (assuming the Einstein field equations), the stress tensor near any such Killing horizon will be highly constrained. Most notably, the sum of the energy density and the transverse pressure will tend to zero as the horizon approached. It should be re-emphasized that such constraints would apply to all forms of matter or energy in the proximity of the horizon; including any quantum-induced fluctuations.

\footnotetext{
${ }^{9}$ The present analysis, however, does not help in computing the actual value of the Einstein tensor. Such a computation would require something like the analysis of the previous section.
} 
Let us recall Carlip's proposal that the black hole entropy is controlled by an asymptotic near-horizon conformal symmetry [13], as this idea served as one of the principal motivations for our work. At first a glance, it may not be evident how our symmetries can be responsible for the type of conformal symmetry that Carlip had in mind. Nevertheless, we would suggest that matter obeying the near-horizon form $T_{\perp} \propto g_{\perp}$ will effectively behave as a collection of world-sheet (two-dimensional) conformal field theories; each of which is defined at a point on the horizon and acts in the transverse plane. As for the parallel block of the stress-tensor, Carlip argues that the physics relevant to leading-order calculations of the black hole entropy should probably be limited to the transverse plane [6].

Important future work should include suitable generalizations to truly dynamical spacetimes. Such generalizations will, however, likely be limited to spacetimes with a high degree of symmetry due to the technically difficult nature of the problem. It should be re-emphasized that if the black hole is evolving slowly enough - on a scale set by the surface gravity - then the near-horizon geometry could be viewed as approximately stationary. (This notion of a dynamical black hole being a quasi-stationary entity is conceptually similar to the isolated horizon framework of Ashtekar et al [28].) It can thus be expected that any of our symmetries remain valid up to corrections that go as the evolution rate.

\section{Acknowledgements}

Research supported by the Marsden Fund administered by the Royal Society of New Zealand, and by the University Research Fund of Victoria University.

\section{References}

[1] J.D. Bekenstein, "Black holes and the second law", Lett. Nuovo Cim. 4, 737 (1972);

"Black holes and entropy", Phys. Rev. D 7, 2333 (1973);

"Generalized second law of thermodynamics in black hole physics", Phys. Rev. D 9, 3292 (1974). 
[2] S.W. Hawking, "Black Hole Explosions", Nature 248 (1974) 30; "Particle creation by black holes", Commun. Math. Phys. 43, 199 (1975).

[3] See, for a relevant discussion,

L. Smolin, "How far are we from the theory of quantum gravity", arXiv:hep-th/0303185 (2003).

[4] A. Strominger and C. Vafa, "The Microscopic origin of black hole entropy", Phys. Lett. B 379, 99 (1996) arXiv:hep-th/9601029.

[5] A. Ashtekar, J. Baez, A. Corichi and K. Krasnov, "Quantum Geometry and Black Hole Entropy", Phys. Rev. Lett. 80, 904 (1998) arXiv:gr-qc/9710007.

[6] S. Carlip, "Black hole entropy from horizon conformal field theory", Nucl. Phys. Proc. Suppl. 88, 10 (2000) arXiv:gr-qc/9912118.

[7] J.L. Cardy, "Operator content of two-dimensional conformally invariant theories", Nucl. Phys. B 270, 186 (1986).

[8] A. Strominger, "Black hole entropy from near-horizon microstates", JHEP 9802, 009 (1998) arXiv:hep-th/9712251.

[9] M. Banados, J. Teitelboim and J. Zanelli, "The black hole in three dimensional space time", Phys. Rev. Lett. 69, 1849 (1992) arXiv:hep-th/9204099.

[10] J.D. Brown and M. Henneaux, "Central charges in the canonical realization of asymptotic symmetries: An example from three-dimensional gravity", Commun. Math. Phys. 104, 207 (1986).

[11] S. Carlip, "Black hole entropy from conformal field theory in any dimension", Phys. Rev. Lett. 82, 2828 (1999) arXiv:hep-th/9812013.

[12] S. Carlip, "Entropy from conformal field theory at Killing horizons", Class. Quant. Grav. 16, 3327 (1999) arXiv:gr-qc/9906126.

[13] S. Carlip, "Near-Horizon conformal symmetry and black hole entropy", Phys. Rev. Lett. 88, 241301 (2002) arXiv:gr-qc/0203001. 
[14] S.N. Solodukhin, "Conformal description of horizon's states", Phys. Lett. B 454, 213 (1999) arXiv:hep-th/9812056.

[15] G. Kang, J.-I. Koga and M.-I. Park, "Near-horizon conformal symmetry and black hole entropy in any dimension", arXiv:hep-th/0402113 (2004).

[16] A.J.M. Medved, D. Martin and M. Visser, "Dirty black holes: Spacetime geometry and near-horizon symmetries", arXiv: gr-qc/0402069 (2004).

[17] M. Visser, "Dirty black holes: Thermodynamics and horizon structure", Phys. Rev. D 46, 2445 (1992) arXiv:hep-th/9203057.

[18] T. Padmanabhan, "Classical and quantum thermodynamics of horizons in spherically symmetric spacetimes", Class. Quant. Grav. 19, 5387 (2002) arXiv:gr-qc/0204019.

[19] M. Visser, "Dirty black holes: Entropy versus area", Phys. Rev. D 48, 583 (1993) arXiv:hep-th/9303029.

[20] M. Visser, "Dirty black holes: entropy as a surface term", Phys. Rev. D D8, 5697 (1993) arXiv:hep-th/9307194.

[21] R.M. Wald, General Relativity, (University of Chicago Press, Chicago, 1984).

[22] I. Racz and R. M. Wald, "Global extensions of space-times describing asymptotic final states of black holes," arXiv:gr-qc/9507055 (1995).

[23] B. Carter, "The general theory of the mechanical, electromagnetic, and thermodynamic properties of black holes", in General Relativity: An Einstein centenary survey, edited by S. W. Hawking and W. A. Israel (Cambridge, England, 1979).

[24] C.W. Misner, K.S. Thorne and J.A. Wheeler, Gravitation (W.H. Freeman, San Francisco, 1973).

[25] See, for instance,

I. Racz, "Does the third law of black hole thermodynamics really have a serious failure?", Class. Quant. Grav. 17, 1353 (2000) arXiv:gr-qc/0009019;

F. Bonjour and R. Gregory, "Comment on Abelian Higgs for extremal 
black holes and selection rules for snapping strings", Phys. Rev. Lett. 81, 5031 (1998) arXiv:hep-th/9809029.

[26] I. Racz and R.M. Wald, "Extension of spacetimes with Killing horizon", Class. Quant. Grav. 9, 2643 (1992).

[27] T. Jacobson, G. Kang and R.C. Myers, "On black hole entropy", Phys. Rev. D 49, 6587 (1994) arXiv:gr-qc/9312023.

[28] A. Ashtekar, A. Corichi and K. Krasnov, "Isolated horizons: The classical phase space", Adv. Theor. Math. Phys. 3, 419 (2000) arXiv:gr-qc/9905089. 